\title{
Intrathecal administration of cyclic AMP response element-binding protein-antisense oligonucleotide attenuates neuropathic pain after peripheral nerve injury and decreases the expression of N-Methyl-D-Aspartic receptors in mice
}

\author{
XIAOPING GU* , JINHUA BO*, WEI ZHANG, XIAOFENG SUN, JUAN ZHANG, \\ YAN YANG and ZHENGLIANG MA \\ Department of Anesthesiology, Affiliated Drum Tower Hospital of Medical Department \\ of Nanjing University, Nanjing, Jiangsu 210008, P.R. China
}

Received February 14, 2013; Accepted March 28, 2013

DOI: 10.3892/or.2013.2437

\begin{abstract}
The aim of the present study was to determine whether the cAMP response element binding protein (CREB) contributes to neuropathic pain during development stage. Adult (7-8 weeks old) male C57BL/6 mice weighing 20-25 g were used. Intrathecal catheter implantation and chronic constriction of the sciatic nerve of the animals were performed. Western blotting and reverse transcription PCR experiments were carried out. Our study demonstrated that the expression of spinal NMDAR after peripheral nerve injury was modulated by central CREB. Chronic constriction nerve injury (CCI) in mice induced thermal hyperalgesia and mechanical allodynia. The increase of NR1 and NR2B subunits of the NMDAR was significantly diminished by intrathecal administration of the CREB antisense oligonucleotide against CREB and pCREB. Additionally, nociceptive behavior induced by CCI was attenuated by intrathecal administration of the CREB antisense oligonucleotide during the period of injection, and the above effects of relieving pain lasted at least 12 days following the last injection. Our results suggested that central functional pCREB may contribute to the development of neuropathic pain and regulate the expression of the NR1 and NR2B subunits of the NMDAR in the process.
\end{abstract}

Correspondence to: Dr Zhengliang Ma, Department of Anesthesiology, Affiliated Drum Tower Hospital of Medical Department of Nanjing University, Nanjing, Jiangsu 210008, P.R. China

E-mail: njgxp1970@163.com

"Contributed equally

Key words: neuropathic pain, cAMP response element-binding protein, NMDAR, intrathecal injection

\section{Introduction}

Neuropathic pain is induced by various kinds of etiologies and share similar clinical characteristics, such as persistent spontaneous pain, hyperalgesia and allodynia. Because of the limited understanding of the mechanisms of neuropathic pain, current therapeutic methods are not ideal. Instead of the taking therapeutic measures after the establishment of the pain, treating it during the development of neuropathic pain might allow patients to have a better prognosis. Therefore, determining the potential mechanisms responsible for the induction of neuropathic pain is required to develop efficacious therapies.

As a constitutive transcription factor, CREB has been proved to be involved in the maintenance of neuropathic pains $(1,2)$, though its specific function is not clear. It has been shown that increased pCREB played an important role in many kinds of pain models, such as inflammation pain $(3,4)$, neuropathic pain $(1,2,5-7)$ and chronic muscle pain $(8)$. What is more, the nociceptive behavior induced by inflammation and neuropathic pain of these models have been shown accompanied with the increase of pCREB $(7,9)$. Ma et al (1) and Wang et al (2) reported that interrupting the induction of pCREB by intrathecal injection of CREB antisense inhibited the peripheral nerve injury-evoked nociceptive behavior in the maintenance of neuropathic pain.

An increasing body of evidence indicates that pCREB is crucial for the transmission of activities occurring at membranes to regulate the expression of downstream genes, such as c-fos (10), c-jun (11), BDNF (brain-derived neurotrophic factor), tyrosine hydroxylase, and many neuropeptides (such as somatostatin, enkephalin and corticotropin-releasing hormone) (12), NR1, NR2B (13-21). Among them, NR1 and NR2B encode two NMDA receptor subunits, which may be regulated by $\mathrm{Ca}^{2+}$-sensitive signaling pathway. Long-term potentiation, learning and memory storage in neurons may rely on $\mathrm{Ca}^{2+}$-influx through NMDA receptors, which act mainly via the induction of calcium-calmodulin kinases and the activation of CREB. Klein et al (13) found that reporter gene driven by the NR2B promoter could be expressed in 
both neuronal and non-neuronal cell lines. NR2B expression is possibly regulated via Sp1 binding sites and a CREB binding site, linked to $\mathrm{Ca}^{2+}$-signaling pathways. The findings of Lau et al (14) also suggested that the transcription of NR1 is regulated by the c-AMP signaling pathway, most likely through CREB and its activation by phosphorylation in cortical neurons. CREB might also be involved in mediating ethanol-induced upregulation of NR2B gene in fetal mouse cortical cells (15). Inhibiting CREB will affect the expression of NR2B (16) and constitutively active CREB can increase both $N R 2 B$ mRNAs and NR2B proteins. The functional CREB regulate learning and long-term plasticity (22-24).

Our previous study showed that NR1 and NR2B are essential in the development and maintenance of neuropathic pain (25-27). Injecting fibrosarcoma cells into the femur could increase the expression of NR2B in the spinal cord accompanied by mechanical allodynia and thermal hyperalgesia. Both are inhibited by the NR2B selective antagonist ifenprodil (28). Evidence from other investigations indicated that the phosphorylation of CREB in neurons may contribute to the plastic changes in the spinal cord and to the development and maintenance of neuropathic pain eventually $(1,2,5,6)$. However, the effect of CREB on the expression of NMDAR in spinal cord in the development of neuropathic pain has not been previously determined. Thus, we proposed that CREB might regulate the expression of NR1 and NR2B within spinal cord and lead to chronic pain. In order to test this hypothesis, we used intrathecal antisense oligonucleotide treatment to reduce the production of CREB proteins as well as the phosphorylated of CREB.

\section{Materials and methods}

Experimental animals. Adult (7-8 weeks old) male C57BL/6 mice weighing 20-25 g were obtained from the Model Animal Research Center of the Nanjing University, housed on a 12-h light/dark schedule with standard rodent chow and water $a d$ libitum at room temperature $\left(21-24^{\circ} \mathrm{C}\right)$. The experimental protocol was approved by the Animal Care and Use Committee at the Medical College of Nanjing University and complied with the guidelines for the use of laboratory animals (10). All efforts were made to minimize animal suffering and to reduce the number of animals used.

Intrathecal catheter implantation. An intrathecal catheter was implanted in each mouse under the same surgical condition. Under deep sodium pentobarbital ( $40 \mathrm{mg} / \mathrm{kg}$, i.p.) anesthesia, the surgical procedures of intrathecal catheterization in this study was based on minor modifications of previous methods (11). After intrathecal catheterization, the mice were allowed to recover for 3 days before being used experimentally. At the experimental day, the mice appeared to be neurologically normal and complete paralysis of the bilateral hind legs after intrathecal administration of $2 \%$ lidocaine $(2 \mu \mathrm{l})$ were used for surgery. The mice were kept in individual cages after catheterization.

Chronic constriction injury (CCI). Male mice were anesthetized with sodium pentobarbital $(40 \mathrm{mg} / \mathrm{kg}$, i.p.) for surgical procedures. Chronic constriction of the sciatic nerve was performed according to the method described by Bennett and Xie (12). Briefly, the right sciatic nerve was exposed at the level of the mid-thigh. Three ligatures (5-0 chromic gut; Ethicon, Rome, Italy) were tied loosely around the sciatic nerve with a $1.0-1.5 \mathrm{~mm}$ interval between each ligature. The wound was then closed with 4-0 Ethicon silk suture in layers. Then, the injured right hind paw was named as ipsilateral paw and the uninjured left hind paw was named as contralateral paw.

Intrathecal CREB antisense ODN administration. The sequences of these sense, missense and antisense CREB ODNs were designed as previously reported (17). Sequences for the ODNs were as follows: antisense, 5'-TGGTCATCTAGTCACCGG TG-3'; sense, 5'-CACCGGTGACTAGATGACCA-3'; and CREB missense, 5'-GACCTCAGGTAGTCGTCGTT-3'. The ODNs were phosphorothioate-modified and synthesized by Sangon Biotechnology Co. (Shanghai, China). The ODNs were reconstituted in saline before administration. The mice were injected intrathecally with saline $5 \mu \mathrm{l}$, sense $5 \mu \mathrm{l} / 5 \mu \mathrm{g}$, missense $5 \mu \mathrm{l} / 5 \mu \mathrm{g}$ and antisense ODN $5 \mu \mathrm{l} / 5 \mu \mathrm{g}$, respectively, every $24 \mathrm{~h}$ for 6 days. The four groups were called NS, A, S and M groups. Finally, the injection was followed by a flush of $5 \mu 1$ of saline.

Mechanical allodynia. Mice were habituated to the behavioral testing conditions daily for 3 days before initiating baseline testing. Each mouse was placed in an individual transparent plastic compartment $(8.5 \times 11.5 \times 14 \mathrm{~cm})$ on a metal mesh floor and allowed to acclimatize for $30 \mathrm{~min}$ each time. Von Frey filaments (Stoelting Co., Wood Dale, IL, USA) with incremental stiffness (0.16-1.4 g) were applied serially to the paw in ascending or descending order of stiffness depending on the foot withdrawal response of the mouse. The maximum and minimum cut-offs were at 1.4 and $0.16 \mathrm{~g}$, respectively. The filaments were poked vertically against the plantar surface with sufficient force to cause slight bending against the paw and held for $4-5 \mathrm{sec}$ with an stimuli interval of $\sim 15 \mathrm{sec}$. A withdrawal of hind paw upon the stimulus (at least three times out of five applications) was considered as a positive response, the paw withdrawal mechanical threshold (PWMT) was determined by sequentially increasing and decreasing the stimulus strength (the up-and-down) method (29). Each mouse was tested five times per stimulus strength. The lowest Von Frey filaments which had three or more positive responses were regarded as PWMT.

Thermal hyperalgesia. Mice were habituated to the behavioral testing conditions for 3 days before initiating baseline testing. Each mouse was placed in an individual transparent plastic compartment $(8.5 \times 11.5 \times 14 \mathrm{~cm})$ on a thin glass platform and allowed to acclimatize for $30 \mathrm{~min}$ each time. A radiant thermal stimulator (Ugo Basile 7370; Plantar Test Apparatus, Comerio, Italy) was placed onto the plantar surface of the hind paw through the glass platform. There were five trials per mouse and 5-min intervals between trials. The infrared intensity was set at 50 (corresponding to $196 \mathrm{~mW} / \mathrm{cm}^{2}$ ), which produced baseline paw withdrawal latencies of 5-10 sec. A cut-off time of $20 \mathrm{sec}$ was used to avoid tissue damage. The mean latency of withdrawal response of each hindpaw was determined by 5 tests. 

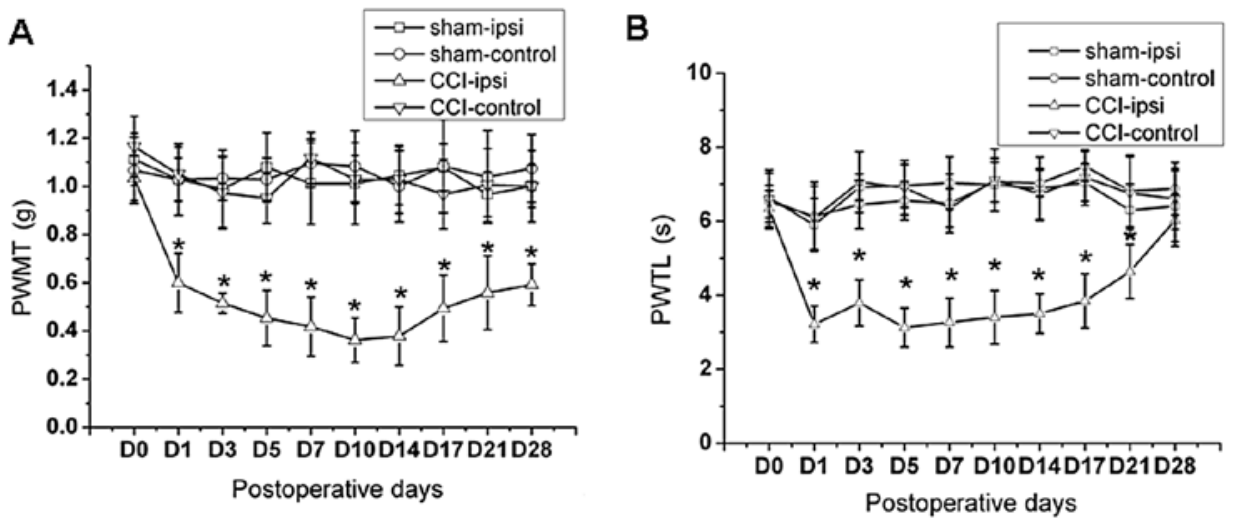

Figure 1. Time course of changes in (A) PWMT and (B) PWTL after CCI. 'ipsi' represents the same side of operation. 'contro' represents the contralateral side of operation. D0, D1, D3, D5, D7, D10, D14, D17, D21 and D28 indicate days of CCI. All data points are means \pm SD. ${ }^{*}<<0.05$, a significant difference as compared with the same side of sham group.

Western blot analysis. Mice were sacrificed rapidly by decapitation and the lumbar spinal cord segments were dissected out and frozen on dry ice in collecting tubes. Samples were then stored at $-80^{\circ} \mathrm{C}$ until further processing. Tissue samples were homogenized in lysis buffer. The homogenate was centrifuged at $12,000 \mathrm{rpm}$ for $10 \mathrm{~min}$ at $4^{\circ} \mathrm{C}$ and supernatant was removed. The protein concentration was determined by the BCA Protein Assay kit, following the manufacturer's instructions. Samples $(50 \mu \mathrm{g})$ were separated on SDS-PAGE (6-12\% gradient gel; KeyGen, Nanjing, China) and subsequently transferred to polyvinylidene difluoride membranes (Millipore Corp., Billerica, MA, USA). The filter membranes were blocked with $5 \%$ non-fat milk in Tris-buffered saline (TBS; $\mathrm{pH} 7.4$; Sigma) for $1 \mathrm{~h}$ at room temperature and incubated respectively with rabbit anti-CREB (1:1,000; Cell Signaling Technology, Inc., Danvers, MA, USA), anti-pCREB (1:1,000; Cell Signaling Technology), anti-NR1 (1:1000; Cell Signaling Technology), anti-NR2B (1:1000; Cell Signaling Technology), and anti- $\beta$ actin (1:2,000, Cell Signaling Technology) primary antibody at $4^{\circ} \mathrm{C}$ overnight. Following three consecutive 5-min washes with TBS, the membrane was incubated with anti-goat or mouse IgG (1:5,000; KeyGen). The blots were visualized in ECL solution (DuPont NEN, Boston, MA, USA) for $1 \mathrm{~min}$ and exposed to hyperfilm (Amersham Biosciences, Piscataway, NJ, USA) for 1-10 min. Immunopositive bands were quantified using Quantity One software (Bio-Rad Laboratories, Hercules, CA, USA). The density of specific bands was measured with a computer-assisted imaging analysis system and normalized against corresponding loading $\beta$-actin bands.

Reverse transcriptase-PCR. Mice were rapidly ( $<1 \mathrm{~min})$ sacrificed through decapitation after being anesthetized with pentobarbital and the L3-L5 lumbar spinal cord segments were immediately frozen in liquid nitrogen and stored at $-80^{\circ} \mathrm{C}$. Previous studies using a retrograde neuronal tracer showed that neurons innervating the distal femur originated from the L3-L5 dorsal root ganglions (DRGs) (18). Total RNA was isolated with TRIzol (15596-026; Invitrogen, Carlsbad, CA, USA), and a $5 \mu \mathrm{g}$ portion of it was used for cDNA synthesis with M-MLV reverse transcriptase (PC0002; Fermentas, Vilnius, Lithuania). The cDNA was used as template for PCR amplification with Taq DNA polymerase (EP0702; Fermentas).
NR1 primers (upstream primer, 5'-ACAACAAGCTGCACGC CTTTA-3' and downstream primer, 5'-TGTTGTTCGACGTG CGGAAAT-3'), NR2B primers (upstream primer, 5'-GTGG G TACGGGAGGGATAGG-3', and downstream primer, 5'-CA CCCATGCCCTCCCTATCC-3') and GAPDH primers (upstream primer, 5'-GAGACCTTCAACACCCCAGC-3', and downstream primer, 5'-CACAGAGTACTTGCGCTCAG-3') designed by Nanjing KeyGen Biological Technology Development Co. The amplified cDNA was electrophoresed on $2 \%$ agarose gel and stained with ethidium bromide. The intensity of each PCR band was analyzed using gel imaging analytical system (Gel Doc XR; Bio-Rad Laboratories). Samples without the addition of reverse transcriptase (negative controls) yielded no detectable product.

Statistical analysis. Western blotting, behavioral and RT-PCR data were analyzed using ANOVA. The post hoc (Student Newman-Keuls) tests were performed to determine sources of differences, when significant main effects were observed. In all cases, $\mathrm{P}<0.05$ is considered to indicate statistically significant result.

\section{Results}

CCI induces pain behavior. Before CCI operation (D0), no differences of the paw withdrawal threshold were observed between sham group (group Sham) and CCI group (group CCI) (Fig. 1, P>0.05). Pain behavior threshold from both sides of hind paw in sham group and the contralateral hind paw in CCI group have no significant change. The paw withdrawal threshold (PWMT) with the strength of the Von Frey filament stimulation of the ipsilateral hind limb significantly decreased after CCI in mice from D1 to D28, and the paw withdrawal threshold latency to thermal stimulation (PWTL) prominently reduced from D1 to D21 (Fig. 1, P<0.05). We considered the behavior test from D0 to D28, because the PWTL recovered to preoperative level on D28.

The expression of pCREB increases after CCI. In CCI, but not in sham group, the expression of $\mathrm{pCREB}$ within the spinal cord increased after peripheral nerve injury (Fig. 2) $(n=3-4)$. The protein level for $\mathrm{pCREB}$ began to increase on postoperative 


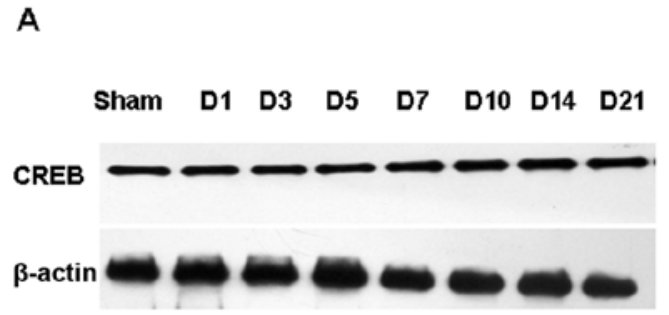

C

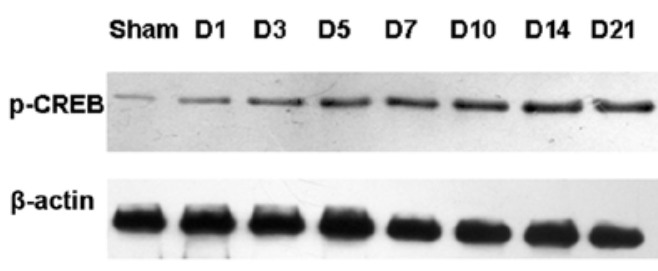

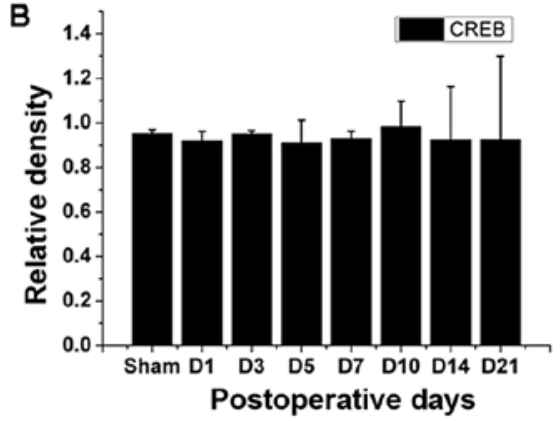

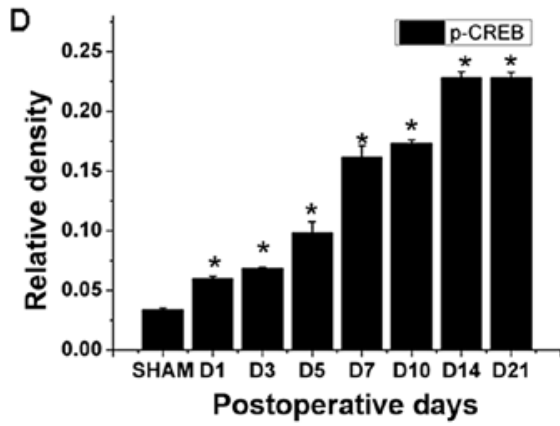

Figure 2. Time course of the CREB and pCREB protein expression and statistical analysis within the spinal cord dorsal horn (n=3-4) after CCI. The L3-L5 spinal cords were collected for analysis after surgery. Proteins were extracted from L3-L5 spinal cord. Representative western blot analysis for CREB (A) and phosphorylation of CREB (C) at the L3-L5 spinal cord are shown. Quantification of (B) CREB and (D) pCREB in each group are shown. Data were obtained from two groups. Data are expressed as means $\pm \mathrm{SD}$. ${ }^{*} \mathrm{P}<0.05$ vs. sham group. The data from day 7 after sham operation are presented as the sham control because there were no differences in the expression of CREB and pCREB in sham mice across postoperative days 1,3, 5, 7, 10, 14 and 21 in our pilot experiments. $\beta$-actin served as a loading control in all panels.
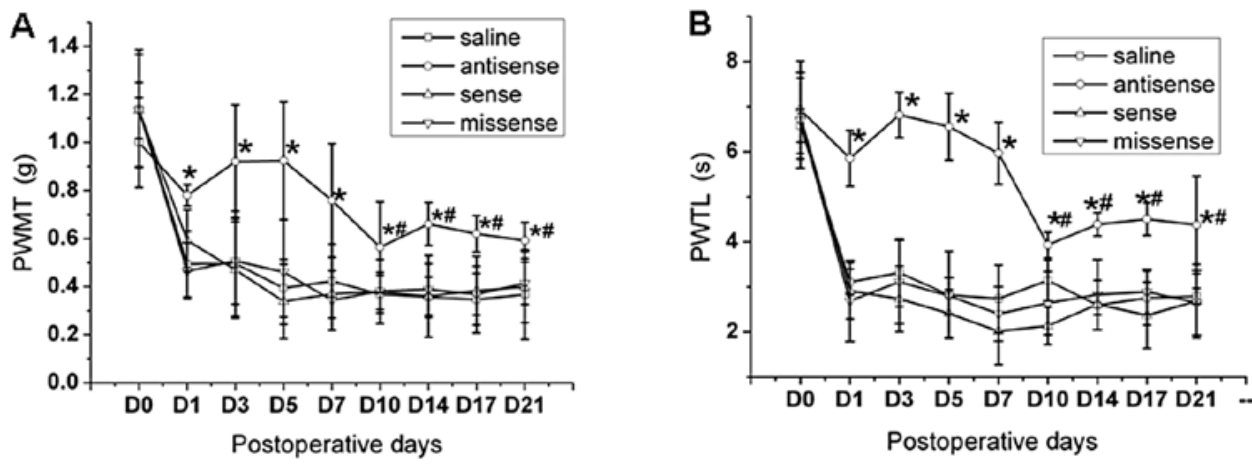

Figure 3. Effects of intrathecal administration of antisense CREB ODN on pain behavior induced by CCI. Tests of the (A) PWMT and (B) PWTL after intrathecal injection of CREB antisense, sense, missense ODN and saline once a day from 1-6 days after CCI. Data were obtained from four groups, with each using 6 mice. D0, D1, D3, D5, D7, D10, D14, D17 and D21 indicate days of CCI. "P<0.05 vs. saline group. " $\mathrm{P}<0.05$ vs. pre-lesion value.

day 1 in CCI mice and remained elevated when examined on postoperative day $21(\mathrm{P}<0.05)$. There was no significant difference in the CREB expression in the spinal cord between CCI and sham mice $(\mathrm{P}>0.05)$ detected by western blot analysis. Thus, CCI induced a time-dependent expression of pCREB at the protein level, but not CREB.

Intrathecal administration of CREB antisense ODN attenuates pain behavior induced by CCI. From 1 to 7 days after intrathecal injection of antisense CREB ODN, the pain behavior in the ipsilateral hindpaw was attenuated to the prelesion value (Fig. 3, P>0.05). From 10 to 21 days after first injection of antisense CREB ODN, the PWMT and PWTL were significantly reduced when compared to pre-lesion value.
However, PWMT and PWTL in the ipsilateral hindpaw of mice with injection of saline, sense and missense CREB ODN remained at regular level at all the time points tested after CCI. The PWMT and PWTL in the ipsilateral hindpaw remained significantly attenuated 10-21 days after first intrathecal injection of antisense CREB ODN, when compared to the other three groups (Fig. 3, $\mathrm{P}<0.05$ ), but was never eliminated to the pre-lesion value.

Intrathecal administration of CREB antisense ODN causes the reduction of expression of $p C R E B$ and CREB. We were interested in examining whether the effect of CREB antisense ODN on allodynia during the development of neuropathic pain actually caused the reduction of CREB or pCREB protein 
A

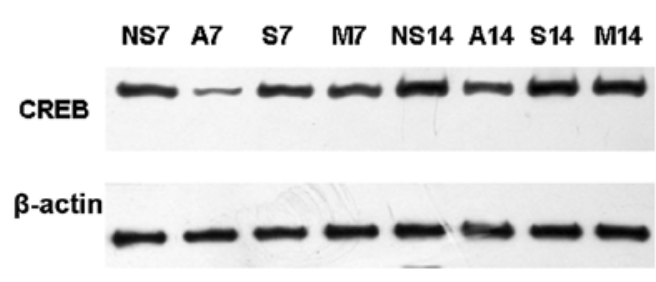

C

$\begin{array}{llllllll}\text { NS7 } & \text { A7 } & \text { S7 } & \text { M7 } & \text { NS14 A14 } & \text { S14 } & \text { M14 }\end{array}$

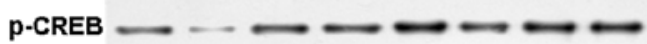

$\boldsymbol{\beta}$-actin

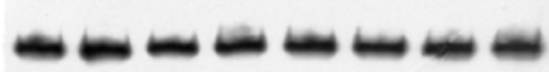

B

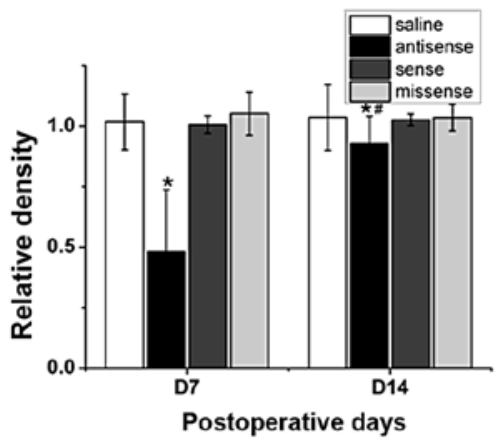

D

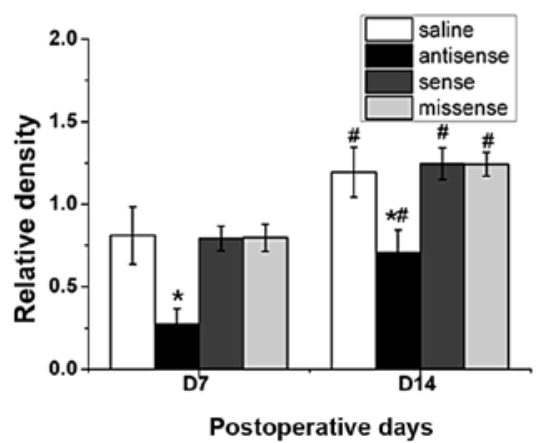

Figure 4. Effects of intrathecal administration of CREB antisense ODN on the expression of pCREB and CREB. The CREB (A and B) and pCREB expression (C and D) were tested in CCI mice treated with the CREB antisense, sense, missense ODN or saline on day 7 and day 14. The mean optical density of CREB and p-CREB at the L3-L5 spinal cord are summarized. Data were obtained from four groups. $\beta$-actin served as a loading control. " $\mathrm{P}<0.05$, a significant difference when compared with saline group. ${ }^{\#} \mathrm{P}<0.05$, significant difference between different time points within a group.

A

$\begin{array}{lllllllll}\text { NS7 } & \text { A7 } & \text { S7 } & \text { M7 } & \text { NS14 } & \text { A14 } & \text { S14 } & \text { M14 }\end{array}$

NR1
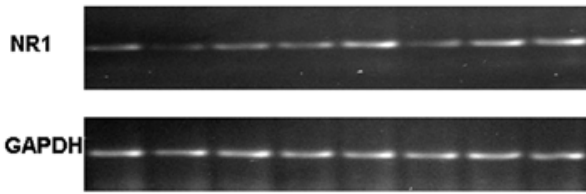

C

$\begin{array}{llllllll}\text { NS7 } & \text { A7 } & \text { S7 } & \text { M7 NS14 } & \text { A14 } & \text { S14 } & \text { M14 }\end{array}$

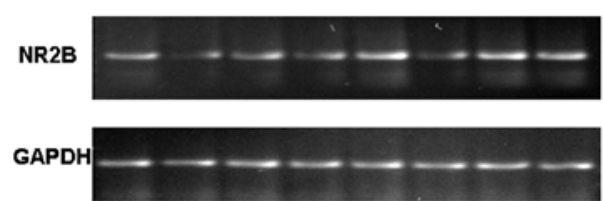

B

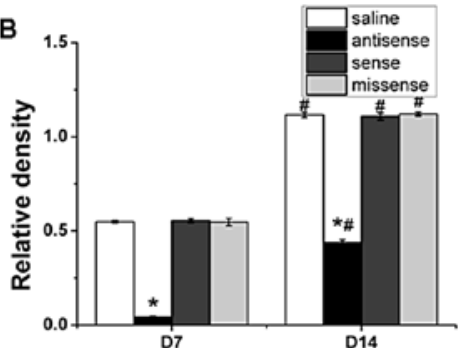

Postoperative days

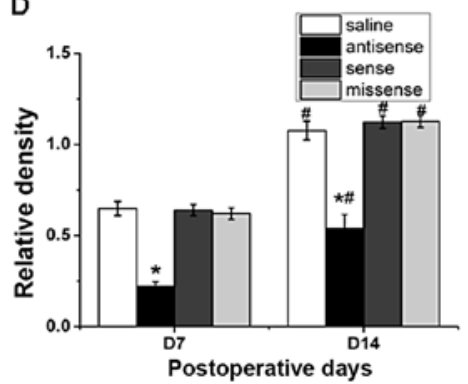

Figure 5. Measurement of NMDAR mRNA levels in spinal cord after intrathecal administration of CREB antisense ODN. The NR1 (A and B) and NR2B (C and D) mRNA expression changes were tested in CCI mice treated with the CREB antisense, sense, missense ODN or saline on day 7 and 14 . The mRNA was extracted from the L3-L5 spinal cord. Data were obtained from four groups. GAPDH is a loading control. * $\mathrm{P}<0.05$, a significant difference when compared with saline group. ${ }^{\#} \mathrm{P}<0.05$, significant difference between different time points within a group.

within the spinal cord. Western blot analysis showed that both the expression levels of CREB and pCREB within the spinal cord were significantly reduced after the application of CREB antisense ODN at day 7 and 14 after CCI compared with control groups (Fig. 4, $\mathrm{P}<0.05$ ). In addition, the expression of CREB and pCREB at day 14 after CCI was higher than the expression at day 7 (Fig. $4, \mathrm{P}<0.05$ ). No significant difference was found between the saline group, CREB sense ODN group and CREB missense ODN group, consistent with previous studies $(1,2)$.

NMDAR mRNA levels in spinal cord are reduced after intrathecal administration of $C R E B$ antisense $O D N$. To investigate whether the interruption of CREB expression would influence 
A

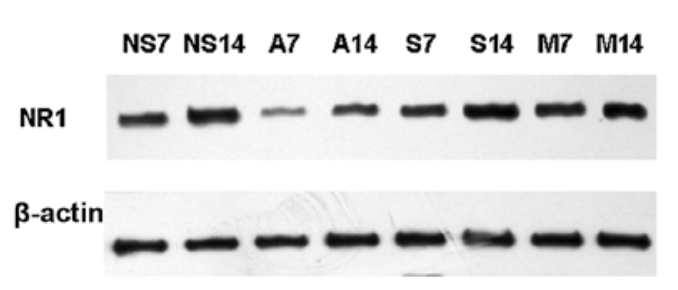

C

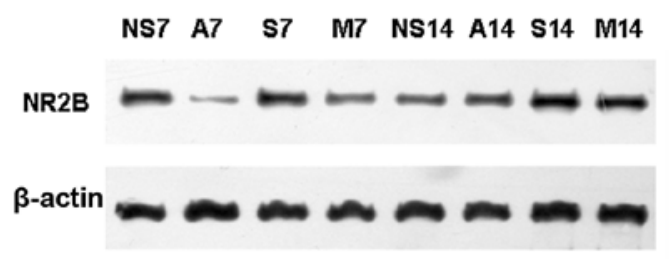

B

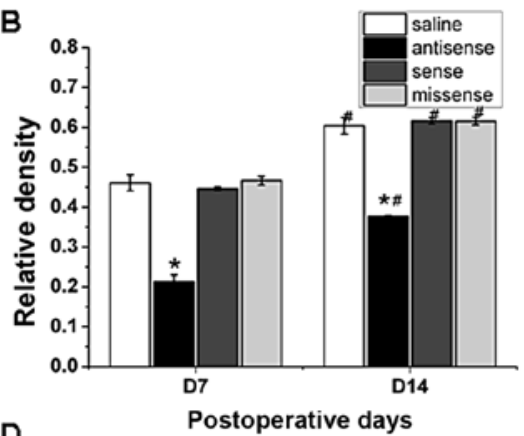

D

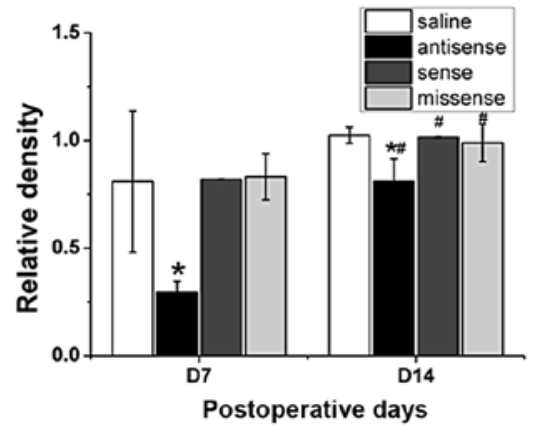

Figure 6. Measurement of NMDAR protein levels in spinal cord after intrathecal administration of CREB ODN. The NR1 (A and B) and NR2B (C and D) protein expression changes were tested in CCI mice treated with the CREB antisense, sense, missense ODN or saline on day 7 and 14 . Proteins were extracted from the L3-L5 spinal cord. Data were obtained from four groups. $\beta$-actin served as a loading control. ${ }^{*} \mathrm{P}<0.05$, a significant difference when compared with saline group. ${ }^{\#} \mathrm{P}<0.05$, significant difference between different time points within a group.

NMDAR mRNA transcription in CCI mice, we conducted reverse transcriptase PCR. In this experiment, each CCI mouse received $5 \mu \mathrm{g} / 5 \mu \mathrm{l}$ a CREB antisense OND, sense OND, missense OND, or saline ( $n=6-8)$, all of which were given once daily via an intrathecal catheter on postoperative days 1 to 6 . The spinal cord samples were harvested on postoperative day 7 and 14 (n=3-4 per group per day). The mRNA levels of NR1 and NR2B within the spinal cord were significantly reduced on postoperative day 7 and 14 in CCI mice after the injection of the CREB antisense OND, compared with the saline group (Fig. 5) $(\mathrm{P}<0.05 ; \mathrm{n}=3-4)$. NR1 and NR2B mRNAs at day 14 within the spinal cord were evidently increased compared to day 7 (Fig. 5) $(\mathrm{P}<0.05$; n=3-4). No significant difference was observed between the saline group, CREB sense ODN group and CREB missense ODN group at each time point.

NMDAR expression increases in spinal cord after intrathecal administration of CREB antisense ODN. To understand whether the interruption of CREB expression would influence NMDAR within spinal cord in CCI mice, we tested the expression level of NMDAR using western blot analysis. In this experiment, each CCI mice received $5 \mu \mathrm{g} / 5 \mu \mathrm{l}$ of CREB antisense OND, sense OND, missense OND, or saline (n=6-8), all of which were given once daily via an intrathecal catheter on postoperative days 1 to 6 . The spinal cord samples were harvested on postoperative day 7 and $14(n=3-4$ per group/day). The expression of NR1 and NR2B subunits within the spinal cord from CCI mice receiving the CREB antisense OND was significantly reduced on postoperative day 7 and 14 compared with the saline group (Fig. 6) $(\mathrm{P}<0.05$; $n=3-4)$. The expression levels of NR1 and NR2B at day 14 within the spinal cord were clearly increased compared to day 7 (Fig. 6) $(\mathrm{P}<0.05 ; \mathrm{n}=3-4)$. The expression of NR2B on day 14 in saline group did not change compared with day 7. No significant difference was found between the saline group, CREB sense ODN group and CREB missense ODN group at each time point.

\section{Discussion}

Many previous studies have suggested that the activation of CREB in the spinal dorsal horn plays an important role in the process of pain induced by inflammation $(3,4,9)$ and nerve injury (5). However, the role of CREB in sensitization of the spinal neurons still remains unclear. It is known that CCI causes neuropathic pain associated with behavioral changes. After CCI, the PWMT of the ipsilateral hind limb was significantly reduced, although the PWTL prominently decreased from D1 to D21 and not as much afterwards. Consistent with the PWTL trend, the expression of the pCREB was activated during the same time period. It is known that pCREB is the functional form which is associated with thermal hyperalgesia and mechanical allodynia after CCI. Our study found that the expression of pCREB increased after CCI while the expression of CREB had no significant changes at that stage. These findings are in line with previous research stating that pCREB in the spinal dorsal horn of rats increases following formalin injection $(4,9)$, carrageenan injection (3), partial sciatic nerve ligation (1,5), chronic constriction injury of the sciatic nerve (6) and spared nerve injury (2). On the basis of previous studies, our study used mice after CCI to study CREB and thus revealed that the pCREB was highly expressed during the whole process of neuropathic pain, suggesting its role in the induction and maintenance of neuropathic pain induced by CCI. 
The present study further demonstrated that chronic daily intrathecal administration of CREB antisense ODN during the development of neuropathic pain alleviated the CCI-induced mechanical allodynia and thermal hyperalgesia possibly due to its function in repressing the expression of CREB in spinal cord of CCI mice. Intrathecal treatment with CREB antisense ODN in the development of neuropathic pain induced by CCI completely relieved pain behavior during the course of injection, while after all the treatment tactile allodynia and thermal hyperalgesia elicited by CCI was attenuated, but not eliminated. Ma et al (1) indicated that 3 weeks following partial sciatic nerve ligation, the tactile allodynia in the ipsilateral hindpaw was significantly attenuated 3-7 days after intrathecal injection of antisense CREB ODN for 5 days, when compared with pre-injection value, but never eliminated to the pre-lesion value and expression of total CREB and pCREB was reduced concomitantly. In another study, Wang et al (2) suggested that chronic injection of CREB antisense ODN for 5 days after 2 weeks following spared nerve injury significantly attenuated SNI-induced mechanical (bilaterally) and cold allodynia (ipsilaterally) at the same time, but never eliminated to the pre-lesion value and also reduced total CREB and pCREB to an almost equal extent in both the ipsilateral and the contralateral dorsal horn neurons. Previous studies suggested that the CREB antisense ODN could not reverse the PWMT and PWTL to pre-lesion value and lasted four days after injection. In our study, we took intrathecal injection of antisense CREB ODN from D1 to D6, once a day. Collectively, these findings suggested that interruption of the expression of CREB by CREB antisense ODN during the induction of neuropathic pain plays a definitively role in the whole process of neuropathic pain.

Once CREB is phosphorylated, it can bind to specific DNA consensus sequences to activate the expression of its downstream genes, such as NR1 and NR2B (NMDA subunits) shown in this study. NMDA receptors contain heteromeric combinations of the NR1 subunit and one or more of NR2A-D subunits (19). Among all subunits, NR1 and NR2B are predominant and their dynamic patterns determine many of the biophysical and pharmacological properties of NMDA receptors (20). Previous studies showed that CREB affects NR1 and NR2B (13-16,21,30-33). It is known that NR1 and NR2B play important roles in neuropathic pain (25-27). Our study revealed that intrathecal injection of CREB antisense after CCI attenuated neuropathic pain by repressing NR1 and NR2B, downstream of CREB.

Zhuo in his study (34) showed that activation of NMDA receptor triggers calcium influx. In adult anterior cingulate cortex (ACC) pyramidal cells, most of NMDA receptors comprise of NR1-NR2A, NR1-NR2B and bits of NR1-NR2ANR2B. $\mathrm{Ca}^{2+}$ influx postsynaptically activates $\mathrm{Ca}^{2+}$-calmodulin (CaM) dependent pathways. Then, $\mathrm{Ca}^{2+}$ and $\mathrm{CaM}$ start to stimulate $\mathrm{AC} 1$ to generate the key second messenger cAMP, which subsequently activates PKA. The catalytic subunit of PKA may relocate to the nucleus and phosphorylate CREB at ser-133 site (35). Phosphorylated CREB activates NR2B gene expression. Subsequently, NR2B is increased, and together with endogenous motor protein KIF17, these new NR2B subunits are added to postsynaptic NMDA receptors. NMDA NR2B receptor-AC1-cAMP-CREB-NR2B might form a posi- tive feedback to reinforce the NMDA receptor functions in the ACC neurons, thus, may further enhance neuronal excitability within the ACC and contribute to chronic pain.

Our study suggested that the NMDA-AC1-cAMP-CREBNMDA loop may function in spinal cord as well. Some results showed that not only NR2 but also NR1 of the NMDAR was activated after CCI in the ipsilateral spinal cord dorsal horn (36). Their mRNA level began to increase at postoperative day 3 in CCI mice and remained elevated when examined on postoperative day 7 and 14. CCI induced a time-dependent and region-specific expression of NMDARs at both the mRNA level and the protein level (36). In our study, we also found that intrathecal injection of CREB antisense inhibited mechanical allodynia and thermal hyperalgesia as well as the increase of NR1 mRNA and protein levels in the spinal cord of mice after CCI. Our results provide novel evidence that increased expression of spinal NMDAR induced by neuropathic pain depends on the activation of the CREB.

Previous studies have suggested that the activation of spinal NMDAR can also potentiate CREB phosphorylation, producing a positive feedback to the hypersensitization of spinal nociceptive neurons (34). In an inflammation model, the NMDAR antagonist of MK-801 inhibits the increased phosphorylation of CREB in the dorsal horn neurons (9). It has been found that the NMDAR antagonist of MK-801 could increase CREM/ICER activity, which opposed CREB $(29,37,38)$. The activation of CREB increases both NMDAR-mediated synaptic currents and surface level of NMDAR, and vice versa, the inhibition of NMDAR abolishes the effect of CREB (32). Our study provides important evidence that the positive feedback of NMDAR NR2B-CREB-NR2B may contribute to the hypersensitization of spinal nociceptive neurons and inhibiting CREB can interrupt this positive feedback.

Collectively, the above data demonstrated that mechanical allodynia and thermal hyperalgesia induced by CCI as well as the upregulation of pCREB expression levels in the spinal cord could be inhibited by intrathecal injection of the CREB antisense ODN. Interrupting the expression of CREB and pCREB during the induction of neuropathic pain could impact in the development of neuropathic pain. Additionally, the lack of pCREB prevented the activation of NR1 and NR2B expression induced by CCI. CREB may be involved in the development of neuropathic pain and regulates expression of NR1 and NR2B subunit of NMDAR in the process. The present study might shed some light on future therapeutic methods on chronic pain.

\section{Acknowledgements}

The present research was supported by the National Natural Foundation of China (30872439/c160202) and (81171047/ H0903).

\section{References}

1. Ma W, Hatzis C and Eisenach JC: Intrathecal injection of cAMP response element binding protein (CREB) antisense oligonucleotide attenuates tactile allodynia caused by partial sciatic nerve ligation. Brain Res 988: 97-104, 2003.

2. Wang YY, Wu SX, Zhou L, Huang J, Wang W, Liu XY and Li YQ: Dose-related antiallodynic effects of cyclic AMP response element-binding protein-antisense oligonucleotide in the spared nerve injury model of neuropathic pain. Neuroscience 139: 1083-1093, 2006. 
3. Messersmith DJ, Kim DJ and Iadarola MJ: Transcription factor regulation of prodynorphin gene expression following rat hindpaw inflammation. Brain Res Mol Brain Res 53: 260-269, 1998.

4. Anderson LE and Seybold VS: Phosphorylated cAMP response element binding protein increases in neurokinin-1 receptorimmunoreactive neurons in rat spinal cord in response to formalin-induced nociception. Neurosci Lett 283: 29-32, 2000.

5. Ma W and Quirion R: Increased phosphorylation of cyclic AMP response element-binding protein (CREB) in the superficial dorsal horn neurons following partial sciatic nerve ligation. Pain 93: 295-301,2001.

6. Miletic G, Pankratz MT and Miletic V: Increases in the phosphorylation of cyclic AMP response element binding protein (CREB) and decreases in the content of calcineurin accompany thermal hyperalgesia following chronic constriction injury in rats. Pain 99: 493-500, 2002.

7. Wang Y, Cheng X, Xu J, Liu Z, Wan Y and Ma D: Antihyperalgesic effect of CaMKII inhibitor is associated with downregulation of phosphorylated CREB in rat spinal cord. J Anesth 25: 87-92, 2011.

8. Hoeger-Bement MK and Sluka KA: Phosphorylation of CREB and mechanical hyperalgesia is reversed by blockade of the cAMP pathway in a time-dependent manner after repeated intramuscular acid injections. J Neurosci 23: 5437-5445, 2003.

9. Ji RR and Rupp F: Phosphorylation of transcription factor CREB in rat spinal cord after formalin-induced hyperalgesia: relationship to c-fos induction. J Neurosci 17: 1776-1785, 1997.

10. Zimmermann M: Ethical guidelines for investigations of experimental pain in conscious animals. Pain 16: 109-110, 1983.

11. Wu WP, Xu XJ and Hao JX: Chronic lumbar catheterization of the spinal subarachnoid space in mice. J Neurosci Methods 133: 65-69, 2004

12. Bennett GJ and Xie YK: A peripheral mononeuropathy in rat that produces disorders of pain sensation like those seen in man. Pain 33: 87-107, 1988

13. Klein M, Pieri I, Uhlmann F, Pfizenmaier K and Eisel U: Cloning and characterization of promoter and 5'-UTR of the NMDA receptor subunit epsilon 2: evidence for alternative splicing of 5'-non-coding exon. Gene 208: 259-269, 1998.

14. Lau GC, Saha S, Faris R and Russek SJ: Up-regulation of NMDAR1 subunit gene expression in cortical neurons via a PKA-dependent pathway. J Neurochem 88: 564-575, 2004.

15. Rani CS, Qiang M and Ticku MK: Potential role of cAMP response element-binding protein in ethanol-induced $\mathrm{N}$-methyl$\mathrm{D}$-aspartate receptor 2B subunit gene transcription in fetal mouse cortical cells. Mol Pharmacol 67: 2126-2136, 2005.

16. Yin X, Takei Y, Kido MA and Hirokawa N: Molecular motor KIF17 is fundamental for memory and learning via differential support of synaptic NR2A/2B levels. Neuron 70: 310-325, 2011.

17. Guzowski JF and McGaugh JL: Antisense oligodeoxynucleotidemediated disruption of hippocampal cAMP response element binding protein levels impairs consolidation of memory for water maze training. Proc Natl Acad Sci USA 94: 2693-2698, 1997.

18. Edoff K, Grenegard M and Hildebrand C: Retrograde tracing and neuropeptide immunohistochemistry of sensory neurones projecting to the cartilaginous distal femoral epiphysis of young rats. Cell Tissue Res 299: 193-200, 2000.

19. Nakanishi S: Molecular diversity of glutamate receptors and implications for brain function. Science 258: 597-603, 1992.

20. Lau CG and Zukin RS: NMDA receptor trafficking in synaptic plasticity and neuropsychiatric disorders. Nat Rev Neurosci 8: 413-426, 2007
21. Bai G and Kusiak JW: Cloning and analysis of the 5' flanking sequence of the rat N-methyl-D-aspartate receptor 1 (NMDAR1) gene. Biochim Biophys Acta 1152: 197-200, 1993.

22. Yin JC and Tully T: CREB and the formation of long-term memory. Curr Opin Neurobiol 6: 264-268, 1996.

23. Mayr B and Montminy M: Transcriptional regulation by the phosphorylation-dependent factor CREB. Nat Rev Mol Cell Biol 2: 599-609, 2001

24. Carlezon WA, Duman RS and Nestler EJ: The many faces of CREB. Trends Neurosci 28: 436-445, 2005.

25. Ma ZL, Zhang W, Gu XP, Yang WS and Zeng YM: Effects of intrathecal injection of prednisolone acetate on expression of NR2B subunit and nNOS in spinal cord of rats after chronic compression of dorsal root ganglia. Ann Clin Lab Sci 37: 349-355, 2007.

26. Gu X, Yang L, Wang S, Sung B, Lim G, Mao J, Zeng Q and Yang C: A rat model of radicular pain induced by chronic compression of lumbar dorsal root ganglion with SURGIFLO. Anesthesiology 108: 113-121, 2008.

27. Zhang W, Shi CX, Gu XP, Ma ZL and Zhu W: Ifenprodil induced antinociception and decreased the expression of NR2B subunits in the dorsal horn after chronic dorsal root ganglia compression in rats. Anesth Analg 108: 1015-1020, 2009.

28. Gu X, Zhang J, Ma Z, Wang J, Zhou X, Jin Y, Xia X, Gao Q and Mei F: The role of N-methyl-D-aspartate receptor subunit NR2B in spinal cord in cancer pain. Eur J Pain 14: 496-502, 2010.

29. Storvik M, Linden AM, Kontkanen O, Lakso M, Castren E and Wong G: Induction of cAMP response element modulator (CREM) and inducible cAMP early repressor (ICER) expression in rat brain by uncompetitive $\mathrm{N}$-methyl-D-aspartate receptor antagonists. J Pharmacol Exp Ther 294: 52-60, 2000.

30. Lonze BE and Ginty DD: Function and regulation of CREB family transcription factors in the nervous system. Neuron 35: 605-623, 2002

31. West AE, Griffith EC and Greenberg ME: Regulation of transcription factors by neuronal activity. Nat Rev Neurosci 3: 921-931, 2002.

32. Huang YH, Lin Y, Brown TE, et al: CREB modulates the functional output of nucleus accumbens neurons: a critical role of $\mathrm{N}$-methyl-D-aspartate glutamate receptor (NMDAR) receptors. J Biol Chem 283: 2751-2760, 2008.

33. Jung WR, Kim HG, Shin MK, Park DI and Kim KL: The effect of ganglioside GQ1b on the NMDA receptor signaling pathway in H19-7 cells and rat hippocampus. Neuroscience 165: 159-167, 2010.

34. Zhuo M: Plasticity of NMDA receptor NR2B subunit in memory and chronic pain. Mol Brain 2: 4, 2009.

35. Gonzalez GA and Montminy MR: Cyclic AMP stimulates somatostatin gene transcription by phosphorylation of CREB at serine 133. Cell 59: 675-680, 1989.

36. Wang S, Lim G, Zeng Q, Sung B, Yang L and Mao J: Central glucocorticoid receptors modulate the expression and function of spinal NMDA receptors after peripheral nerve injury. J Neurosci 25: 488-495, 2005

37. Storvik M, Tiikkainen P, van Iersel M and Wong G: Distinct gene expression profiles in adult rat brains after acute MK-801 and cocaine treatments. Eur Neuropsychopharmacol 16: 211-219, 2006.

38. Konopka D, Szklarczyk AW, Filipkowski RK, Trauzold A, Nowicka D, Hetman M and Kaczmarek L: Plasticity- and neurodegeneration-linked cyclic-AMP responsive element modulator/inducible cyclic-AMP early repressor messenger RNA expression in the rat brain. Neuroscience 86: 499-510, 1998. 